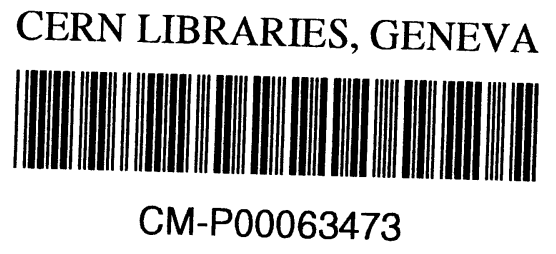

\title{
Top quark physics at the LHC
}

\author{
Peter Uwer ${ }^{\text {a }}$ \\ a Department of Physics, Theory Unit, CERN, CH-1211 Geneva 23, Switzerland \\ In this article I review some aspects of the expected top quark physics at the LHC.
}

\section{Introduction}

The top quark is the heaviest known elementary particle so far. With a mass of $174.3 \pm 3.4 \mathrm{GeV}$ [1] the top quark is almost as heavy as a gold atom. While the top quark mass was already predicted from indirect measurements at LEP, first evidence of the direct observation of the top quark itself was obtained only ten years ago at Fermilab [2,3]. Since then much progress has been obtained; in particular, the error on the top mass went down from more than $10 \mathrm{GeV}$ for the first observations to the aforementioned $3.4 \mathrm{GeV}$. In the Standard Model the top quark completes the third flavour family. As a consequence the quantum numbers of the top quark are completely fixed by the structure of the Standard Model. As up-type partner of the bottom quark the electric charge is $+2 / 3$. The left-handed component has weak ispspin $+1 / 2$, while the right-handed component has weak isospin 0 . So far our knowledge on these quantum numbers is only from indirect measurements. In particular, such a fundamental quantity as the electric charge of the top quark has not been measured yet [4]. In Ref. [5] for example a scenario is discussed where the particle discovered at the Fermilab collider is an exotic quark with charge $-4 / 3$.

Owing to its large mass, the top quark is the only fermion that couples with a strength of order one to the Higgs boson. Furthermore the top mass is close to the scale of electroweak symmetry breaking. The top quark is thus a natural tool to study the electroweak symmetry breaking in more detail. The large mass makes it also an ideal laboratory to search for new physics.

Apart from the interest in top quark physics as signal process, it also plays an important rôle as background in many Higgs studies. In addition, it turns out that top quark physics might also be a very helpful tool in calibrating the detectors. Although many interesting studies can be done already at Fermilab, the DO and CDF experiments are still limited by the small number of top events. With an expected production rate of the order 8 million top quark pair events per year (running at 'low' luminosity) top quark physics at the LHC is in general not limited by statistics. For the near future, the LHC thus offers the unique possibility for precise measurements in the top sector.

In the following I will discuss a few examples for the top as commissioning tool, as background and as signal process.

\section{Top quark as comissioning tool}

If it is assumed that the top quark behaves as predicted by the Standard Model, top quark physics is an interesting tool for calibrating the LHC detectors in the LHC start-up phase. In Ref. [6] it was shown for example that using only very simple cuts, in particular

$$
\begin{aligned}
& \text { - one isolated electron or muon } \\
& \text { with } p_{T}>20 \mathrm{GeV} \\
& \text { - four and only four jets with } p_{T}>40 \mathrm{GeV} \text {, }
\end{aligned}
$$

a $t \bar{t}$-enriched event sample can be obtained. Such an event sample can then be used to study for example the $b$-tagging efficiency or the jet energy scale. Both quantities are crucial for many physics analyses at the LHC. It was also pointed out in Ref. [6] that in difference to the LHC at the Tevatron the top cannot be used for calibration purposes because of the limited statistics.

\section{Top quark as background}

The most important objective of the LHC is to discover the Higgs boson if it exists and to measure its 
properties. In many cases it turns out that a dominant background for the Higgs studies is given by top quark events. For example if the Higgs mass lies in the range $120-190 \mathrm{GeV}$ the most important discovery channel is weak boson fusion. Here the dominant background is given by $t \bar{t}+1$ jet. It is evident that a detailed knowledge of this background is of crucial importance. At the moment, two different strategies are under consideration. One possibility is to determine the background through an extrapolation from the data. This was studied in great detail in Ref. [7], where it was found that an accuracy of 5-10\% might be feasible. The second strategy is the calculation of the next-to-leading order corrections for $t \bar{t}+1$ jet. This is a very challenging calulation, which is currently under way [8]. When the next-to-leading order corrections are available, it will be interesting to compare the two methods, to see how the extrapolation from the data is working. Top quark pair production together with a jet might also be interesting to learn something about $t \bar{t} b \bar{b}$, which is an important background process for $t \bar{t} H$, which can be used to measure the Yukawa coupling of the Higgs to the top quark.

\section{Top quark as signal}

As mentioned in the introduction our knowledge about the top quark from direct measurements is still rather limited. It is thus important to setup an extensive experimental (and theoretical) programme to measure the properties of the top quark at the LHC. Important observables in this context are:

1. Total cross section for top quark pair production, $t \bar{t}$ invariant mass spectrum $(\rightarrow$ mass determination, search for new physics in the $t \bar{t}$ invariant mass spectrum, comparison with theoretical predictions)

2. W polarization in top decay $(\rightarrow$ test of the $\mathrm{V}-\mathrm{A}$ structure, search for anomalous $t W b$ couplings)

3. $t \bar{t} H$ cross section ( $\rightarrow$ Yukawa coupling)

4. single top production $(\rightarrow \mathrm{CKM}$ matrix element $\left.V_{t b}\right)$

5. spin correlation of top quark pairs $(\rightarrow$ search for anomalous $t \bar{t} g$ couplings, decay of a quasi free quark)
6. $t \bar{t} \gamma$ cross section $(\rightarrow$ electric charge of the top quark)

7. $t \bar{t}+1$ jet $(\rightarrow$ search for anomalous $t \bar{t} g$ couplings, jet physics with heavy quarks, important background reaction).

In parenthesis I quoted why a given observable might be interesting to look at. For the observables 1-6 the theoretical prediction is at next-to-leading order accuracy. The calculation of the next-to-leading order corrections to 7 is an ongoing project [8], results are expected for the end of this year. I cannot comment here on all the aforementioned observables. In the following I will discuss in some detail the spin correlations [9-14], because here some progress has recently been made on the theoretical as well as on the experimental side [15-17].

\subsection{Spin correlation of top quark pairs}

Related to the large mass of the top quark is the large width of the top quark. From the leading-order electroweak formula, including QCD corrections, one obtains a width of $\sim 1.42 \mathrm{GeV}$. This leads to a lifetime of $0.44 \times 10^{-24} \mathrm{~s}$. In other words, the top quark decays on average before it has time to hadronize [18]. This is a unique feature. All the lighter quarks form bound states, which may then decay. An important consequence of the fact that the top quark decays as a quasi-free quark is that its spin properties are transferred to the decay products and are not diluted by hadronization, as is the case for the lighter quarks. This offers the possibility to study the polarization of the top quark through the angular distribution of the decay products of the parity-violating decay $t \rightarrow W b-$ the dominant decay channel in the Standard Model. At hadron colliders, the dominant production mechanisms are quark-antiquark annihilation and gluon fusion. While at the Tevatron $\sim 85 \%$ of the $t \bar{t}$ pairs are produced in quark-antiquark annihilation, the situation at the LHC is reversed. Here the dominant production mechanism is gluon fusion. About $85 \%$ are produced in gluon fusion, while the remaining $15 \%$ are produced in quark-antiquark annihilation. (The precise values depend on the factorization/renormalization scale as well as on the chosen parton distribution function.) In both cases the top quarks are produced essentially unpolarized, because of the parity invariance of QCD. (There is a tiny polar- 
ization transverse to the scattering plane, which is induced by absorptive parts at the one-loop level [19].) Although unpolarized, the spins of the top quark and the top antiquark are correlated. At threshold this is very easy to understand. For the $q \bar{q}$-initiated process the top quark pair is in a ${ }^{3} S_{1}$ state. In the centre-ofmass system, where the top and the antitop are back to back, the spins are thus aligned with respect to the helicity basis. For the gluon fusion process the top quark pair is in a ${ }^{1} S_{0}$ state. Using again the helicity basis, the spins are thus opposite to each other. Away from the threshold this simple picture is slightly changed. At this point it is worth keeping in mind that top quark pairs are in general produced at the LHC not far away from theshold. In any case the corrections to the simple picture described above can be calculated. As mentioned before the spin properties can be analysed by studying the angular distributions of the decay products. In particular an observable sensitive to the spin correlation is given by

$$
\frac{1}{\sigma} \frac{d \sigma}{d \cos \left(\theta_{1}\right) d \cos \left(\theta_{2}\right)}=\frac{1}{4}\left(1-C \cos \left(\theta_{1}\right) \cos \left(\theta_{2}\right)\right),(1)
$$

where $\theta_{1}\left(\theta_{2}\right)$ is the angle between the direction of flight of one of the decay products of the top (anti)quark and an arbitrary chosen axis taken in the top (anti)quark rest frame. It can be shown that this arbitrary chosen axis plays essentially the rôle of the quantization axis. The value of $C$ depends on the spin correlation $A$ of the top quark pair, where $A$ is defined by

$$
A=\frac{\sigma(\uparrow \uparrow)+\sigma(\downarrow \downarrow)-\sigma(\uparrow \downarrow)-\sigma(\downarrow \uparrow)}{\sigma(\uparrow \uparrow)+\sigma(\downarrow \downarrow)+\sigma(\uparrow \downarrow)+\sigma(\downarrow \uparrow)} .
$$

Furthermore $C$ depends also on the quality $\kappa$ with which the decay products analyse the top spin:

$\frac{d \Gamma}{\Gamma}=\frac{1}{2}(1+\kappa \cos (\alpha))$.

In leading order, the relation for $C$ is given by

$$
C=\kappa_{1} \kappa_{2} A \text {. }
$$

In addition to the observable above the distribution

$\frac{1}{\sigma} \frac{d \sigma}{d \cos \varphi}=\frac{1}{2}(1-\mathrm{D} \cos \varphi)$,

where $\varphi$ denotes the angle between the direction of flight of the lepton $\ell^{+}$(or jet $j_{1}$ ) and of $\ell^{\prime-}$ (or $j_{2}$ ), defined in the $t$ or $\bar{t}$ rest frames, is also sensitive to the spin correlation. As discussed already, the actual value of $C$ depends on the axis chosen to define the angles $\theta_{1}$ and $\theta_{2}$. In Tables 1 and 2 theoretical predictions are shown for different quantization axes. It is clearly visible that, in general, spin correlations can be large.

\begin{tabular}{|ccccc|}
\hline & & Dilepton & Lepton-jet & Jet-jet \\
\hline $\mathrm{C}_{\text {hel }}$ & LO & -0.471 & -0.240 & -0.123 \\
& NLO & -0.352 & -0.168 & -0.080 \\
\hline C $_{\text {beam }}$ & LO & 0.928 & 0.474 & 0.242 \\
& NLO & 0.777 & 0.370 & 0.176 \\
\hline$C_{\text {off }}$ & LO & 0.937 & 0.478 & 0.244 \\
& NLO & 0.782 & 0.372 & 0.177 \\
\hline D & LO & 0.297 & 0.151 & 0.0773 \\
& NLO & 0.213 & 0.101 & 0.0480 \\
\hline
\end{tabular}

Table 1

$L O$ and NLO results for the spin correlation coefficients $\mathrm{C}$ and $\mathrm{D}$ of the distributions (1) and (5) in the case of $p \bar{p}$ collisions at $\sqrt{s}=1.96 \mathrm{TeV}$ for different $t \bar{t}$ decay modes. The PDF CTEQ6L (LO) and CTEQ6.1M (NLO) of Ref. [20] were used, and $\mu_{F}=$ $\mu_{R}=m_{t}$. The 'off-diagonal' axis used in $C_{\mathrm{off}}$ is defined in [14].

\begin{tabular}{|ccccc|}
\hline & & Dilepton & Lepton-jet & Jet-jet \\
\hline C $_{\text {hel }}$ & LO & 0.319 & 0.163 & 0.083 \\
& NLO & 0.326 & 0.158 & 0.076 \\
\hline D & LO & -0.217 & -0.111 & -0.0567 \\
& NLO & -0.237 & -0.115 & -0.0560 \\
\hline
\end{tabular}

Table 2

Results for $\mathrm{C}_{\mathrm{hel}}$ and $\mathrm{D}$ for $p p$ collisions at $\sqrt{s}=$ $14 \mathrm{TeV}$ using the same PDF and parameters as in Table 1 .

We note that the $C$ has a different sign at the Tevatron with respect to the LHC when using the helic- 
ity basis. This reflects the fact that in $q \bar{q}$ annihilation the spins tend to be aligned while in gluon fusion the spins tend to be opposite to each other. Furthermore a strong dependence on the choice of the quantization axis can be observed for the Tevatron. In particular an almost optimal axis for which the correlation is almost $100 \%$ can be found. This is because, for the $q \bar{q}$ initiated process, an axis yielding $100 \%$ can be constructed [14]. For the LHC where the dominant process is gluon fusion no such axis exists. The helicity basis gives a correlation of around $30 \%$, the beamaxis and the off-diagonal axis give only tiny correlations. At the LHC the maximal value that can be obtained for $C$ is $48 \%$ [21]. In Tables 1 and 2 the next-lo-leading order corrections are also shown. In general the corrections are only of moderate size. In particular at the LHC the corrections are only of the order of a few per cent. In Ref. [16] also the scale dependence was shown to be remarkably small. The good perturbative behaviour can be understood from the fact that the definition for $C$ is actually a ratio of two cross sections. To a certain extent many corrections just cancel in this ratio. Recently the Atlas collaboration performed a detailed analysis to investigate the experimental observation of the spin correlation. In particular the double-leptonic channel (where both top quarks decay into leptons) and the semileptonic channel (one top decays into leptons, the other top into hadrons) were studied [17]. In particular it was found that $C$ can be measured with an uncertainty of only $7 \%$. For $D$ the situation is even better. Here an accuracy of $5 \%$ might be feasible. In both case the semileptonic and the dileptonic channels were combined. The whole analysis was based on $10 \mathrm{fb}^{-1}$ of accumulated luminosity. In most cases the analysis was limited by systematics and not by the statistics. Furthermore it was shown that the same event sample can also be used to measure the $W$ polarization in $t \bar{t}$ events. Here an accuracy at the level of $1-2 \%$ can be achieved.

\section{Conclusion and outlook}

Top quark physics will play an important rôle at the LHC. It can be used for example to calibrate the LHC detectors in the start-up phase. Furthermore the top is for many reactions the dominant background. A precise understanding of top quark physics is thus important. Treating the top as signal process, many interesting measurements can be made at the LHC.

Acknowledgement: I would like to thank the organizers for the pleasant atmosphere at QCD05.

\section{REFERENCES}

1. L. Scodellaro, these proceedings,

2. CDF, F. Abe et al., Phys. Rev. Lett. 74 (1995) 2626, hep-ex/9503002,

3. D0, S. Abachi et al., Phys. Rev. Lett. 74 (1995) 2632, hep-ex/9503003,

4. U. Baur, M. Buice and L.H. Orr, Phys. Rev. D64 (2001) 094019, hep-ph/0106341,

5. D. Chang, W.F. Chang and E. Ma, Phys. Rev. D59 (1999) 091503, hep-ph/9810531,

6. F. Gianotti and M.L. Mangano (2005), hep$\mathrm{ph} / 0504221$,

7. N. Kauer, Phys. Rev. D70 (2004) 014020, hep$\mathrm{ph} / 0404045$,

8. A. Brandenburg et al., Nucl. Phys. Proc. Suppl. 135 (2004) 71, hep-ph/0408137,

9. T. Arens and L.M. Sehgal, Phys. Lett. B302 (1993) 501,

10. J.H. Kühn, Nucl. Phys. B237 (1984) 77,

11. W. Bernreuther and A. Brandenburg, Phys. Rev. D49 (1994) 4481, hep-ph/9312210,

12. G. Mahlon and S.J. Parke, Phys. Rev. D53 (1996) 4886, hep-ph/9512264,

13. T. Stelzer and S. Willenbrock, Phys. Lett. B374 (1996) 169, hep-ph/9512292,

14. G. Mahlon and S. Parke, Phys. Lett. B411 (1997) 173, hep-ph/9706304,

15. W. Bernreuther et al., Phys. Rev. Lett. 87 (2001) 242002, hep-ph/0107086,

16. W. Bernreuther et al., Nucl. Phys. B690 (2004) 81, hep-ph/0403035,

17. F. Hubaut et al. (2005), hep-ex/0508061,

18. I.I.Y. Bigi et al., Phys. Lett. B181 (1986) 157,

19. W. Bernreuther, A. Brandenburg and P. Uwer, Phys. Lett. B368 (1996) 153, hep-ph/9510300,

20. J. Pumplin et al., JHEP 07 (2002) 012, hep$\mathrm{ph} / 0201195$,

21. P. Uwer, Phys. Lett. B609 (2005) 271, hep$\mathrm{ph} / 0412097$. 